\title{
An Overview on Vancomycin Resistant Enterococcus faecalis
}

\author{
*Kumurya, A.S. (D) 19and Ega, B, ${ }^{2}$ \\ ${ }^{1}$ Department of Medical laboratory Science Faculty of Allied Health SciencesBayero \\ University Kano. Nigeria \\ 2 Department of Medical Microbiology ABU, Zaria. Kaduna State \\ ${ }^{*}$ Correspondence author; A. S Kumurya, 07032153561
}

\begin{abstract}
There are over 15 species of the Enterococcus genus, $80-90 \%$ of clinical isolates as $\mathrm{E}$. faecalis. The aim of this work is to review the current information on Vancomycin resistant Enterococcus fecalis. The study reviewed using electronic documents and hard copies from public libraries of relevant literatures relating to biology, epidemiology, drug resistance mechanism, treatment, and control of Enterococcus faecalis. The review revealed that Enterocuccus faecalis formerly known as Streptococcus faecalis is a Gram-positive commensal bacterium that inhabits the gastrointestinal tracts of healthy humans and other mammals. However, it can cause lifethreatening infections in humans, especially in the nosocomial environment, where there are naturally high levels of antibiotic resistance. Thus, Enterococci have proven to present a therapeutic challenge because of their resistance to many antimicrobial drugs, including cell-wall active agents; aminoglycosides, penicillin, ampicillin, and vancomycin." The Enterococci have the capacity to acquire a wide variety of antimicrobial resistance factors through plasmid transfer by conjugation, which present serious problems in the management of patients with Enterococcal infections. In general, Enterococcal isolates with lowered susceptibility to vancomycin are categorized as vanA, vanB, and $\operatorname{vanC}$, vanA and $\operatorname{vanB}$ pose the greatest threat because they are the most resistant genes. E. faecalis are also resistant to teicoplanin. Enterococcal strains that are vancomycin-dependent have been found, but are rare and less common than vancomycin-resistant strains (referred to as "vancomycin-resistant Enterococci" or "VRE"). The review, identified that although VRE infection possess the tendency to become endemic especially in very ill debilitated patients who have been exposed to broad spectrum antibiotics; and the immune-compromised, yet Vancomycin continues to be the drug of choice for serious life threatening infections as sepsis, pneumonia, and endocarditis.

Keywords: Vancomycin-resistant Enterococci(VRE), Enterococcus faecalis, Resistance gene,
\end{abstract}

\section{INTRODUCTION}

Enterococcus faecalis formerly classified as Streptococcus faecalis, is a Gram-positive, commensal bacterium (Jaremko, 2013; Anderson et al., 2015). E faecalis is found in healthy humans, but can cause life-threatening infections in humans, especially in the nosocomial environment. The naturally high levels of antibiotic Resistance found in $E$. faecalis contribute to its pathogenicity. $E$ faecalis is a non-motile microbe; it ferments glucose without gas production and does not produce a catalase reaction with hydrogen peroxide. It can produce a pseudo-catalase response if grown on blood agar. The reaction is usually weak. It produces a reduction of litmus milk, but does not liquefy gelatin. It shows consistent growth throughout nutrient broth which is consistent with being a facultative anaerobe. It survives very harsh environments including highly alkaline $\mathrm{PH}$ (9.6) and salt concentrations. It resists bile salts, detergent, heavy metals, ethanol, azide, and desiccation. It can grow in range of 10 to $45^{\circ} \mathrm{C}$ and survive at temperature of $60^{\circ} \mathrm{C}$ for $30 \mathrm{~min}$ (Anderson et al., 2015). Several virulence factors are thought to contribute to $E$ faecalis survival and infection this is because it endures a prolonged period of nutritional deprivation, alters host responses, suppresses the action of lymphocytes, possesses lytic enzymes, cytolysin, aggregation substance, pheromones, and lipoteichoic acid, it also utilizes serum as a nutritional source, resists intracranial medicaments, competes with other cells and lastly forms a biofilm (Arias et al., 2010). Enterococci are currently leading nosocomial pathogens, becoming the second most common organisms recovered from the nosocomial urinary tract and wound infections. It is the third most common cause of nosocomial bacteremia in the United States (Ceci et al., 
2015; Aliya et al., 2021). One of the significantprimary reasons why these organisms have survived in the hospital environment is their intrinsic Resistance to several commonly used antibiotics and, perhaps more important, their ability to acquire Resistance to all currently available antibiotics, either by mutation or by receipt of foreign genetic material through the transfer of plasmids and transposons (CDC, 2017; Cetinkaya et al., 2000). Vancomycin a glycopeptide antibiotic was first isolated from Amycolatopsis Orientalis (Streptomyces Orientalis) in 1953 by Edmund knornfeld (working at Eli Lilly) from a soil sample collected from the interior jungle of borneo by a missionary (Chuang et al., 2010). The initial indication for Vancomycin was to treat penicillin resistant Staphylococcus aureus (Puzuki et al., 2014). Vancomycin act by inhibiting cell wall synthesis in gram positive bacteria, it is use in the treatment of serious, life-threatening infection by gram positive bacteria not responding to other antibiotics (Cetinkaya et al., 2000; CDC, 2017). In 1988, Uttley et al. were the first to report the isolation of vancomycin-resistant $E$. faecalis and $E$. faecium in England (Cetinkaya et al., 2000; Coombs et al., 2014). Shortly after the first isolates of vancomycin-resistant enterococci (VRE) were reported by investigators in the United Kingdom and France (Cetinkaya et al., 2000; Dance, 2013) similar strains were detected in hospitals located in the eastern half of the United States. Subsequently, VRE spread with surprising rapidity and are now encountered by hospitals in most countries.

\section{MATERIAL AND METHODS}

A comprehensive literature search of studies publish until December, 2017 was performed using the PubMed, Medline, and science Direct databases. The search was strictly limited to full articles in English and studies related to humans. The following key-words were used to extract the relevantarticles relating to biology, epidemiology, drug resistance mechanism, treatment and control of Enterococcus faecalis.
Cross-referncing was also looked for and duly cited in the review process.

Literature review through the electronic search of databases produces a total of 605 articles, including both review and original research articles. Thorough screening gave rise to the selection of 252, which are mostly related to different on human Vancomycin Resistance Enterococcus faecalis. Further screening leads to the rejection of 201 studies, and 51 articles were finally adopted which were strictly related to biology, epidemiology, drug resistance mechanism, treatment and control of Enterococcus faecalis

History of Enterococcus faecalis

Until 1984, Enterococcus faecalis was known as Streptococcus faecalis. Scientists previously categorized the bacteria as part of the genus Streptococcus (Dance, 2013; Jaremko, 2013; Susan, 2018). E. faecalis is a Gram-positive bacterium there are three main components that make up its cell wall: peptidoglycan, teichoic acid, and polysaccharide. $40 \%$ of the cell wall is made up of peptidoglycan, while the rest of the cell wall is made up of a "rhamnosecontaining polysaccharide and a ribitolcontaining teichoic acid" (Flannery, 2011). The peptidoglycan functions (as in most Grampositive cells) to resist bursting induced by high cytoplasmic osmotic pressure $E$. faecalisis generally considered a non-capsulated organism, shown by the "lack of a detectable mucoid phenotype" (Flannery, 2011). However, subsets of $E$. faecalis isolates possess a capsular polysaccharide.

\section{Biofilm Formation}

E. faecalis also can make surface pili which can lead to the formation of a biofilm. The $E$. faecalis strains that cause endocarditis contain large amounts of these pili. The pili allow for attachment to host surfaces (e.g. the heart tissue). The strains of $E$. faecalis that cause endocarditis produce the "biofilm significantly more often and also to a greater degree than non-endocarditis isolates" (Hayakawa et al., 2011). 


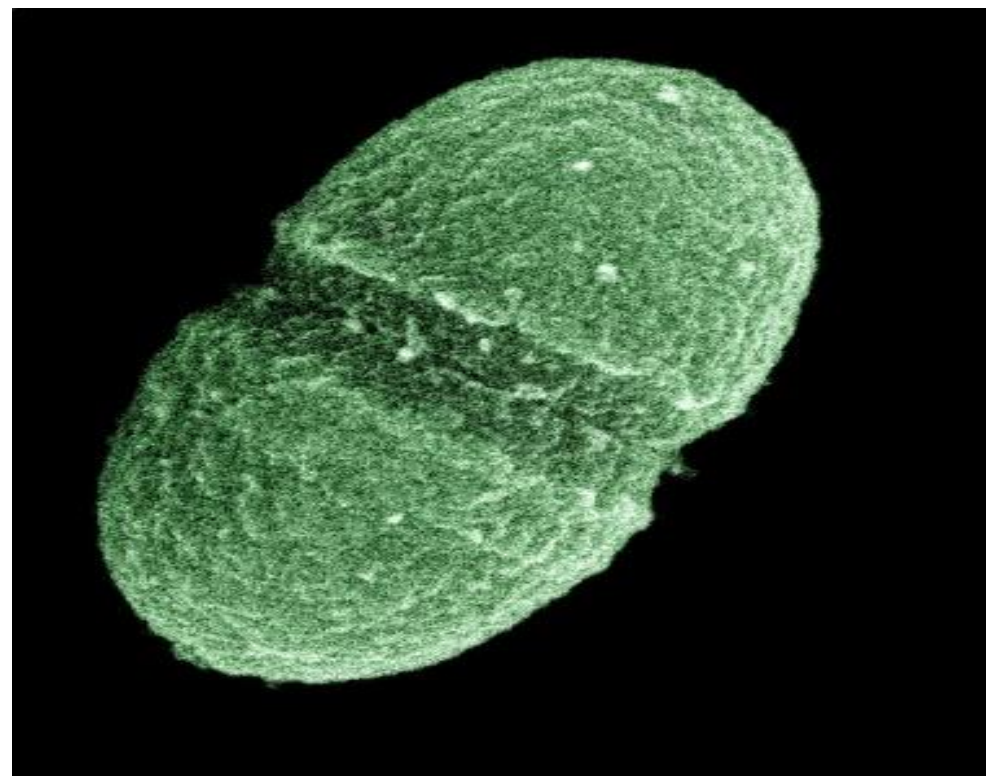

Figure 1: Biofilm formation in enterococcus faecalis

Source:Andrew and Horder, (1906); Schleifer and Kilpper-Balz, (1984).

\section{Genome Structure}

The $E$. faecalis genome consists of 3.22 million base pairs with 3,113 protein-coding genes (Jaremkoet al., 2013). Due to many public health dangers, the genome sequence data from a strain of Enterococcus was necessary. The strain chosen for genome DNA sequencing was $E$. faecalis V583, the first vancomycinresistant isolate in the United States. The genome of strain V583 was sequenced by The Institute for Genome Research (TIGR). The enterococcal genome shows $E$. faecalis is metabolically diverse and contains a wide range of regulatory systems. Strain V583 contains four DNA molecules: the main 3,218,030 base pair bacterial chromosome and three circular plasmids. The chromosome contains about 3,500 open reading frames (ORFs), about $1 / 3$ of these ORFs have no assignable function (Zhu et al., 2010). The three plasmids are circular DNA molecules identified as Plasmid-1, Plasmid-2, and Plasmid-3. Plasmid-1 contains 66,320bp, Plasmid-2 contains $17,963 \mathrm{bp}$, and Plasmid-3 contains $57,660 \mathrm{bp}$. The plasmids encode a number of genes, including, transposases, multi-drug resistance proteins, and a ppGppregulated growth inhibitor. The average $\mathrm{G}+\mathrm{C}$ composition of the $E$. faecalis chromosome is 37.38\% (Anderson et al., 2015).

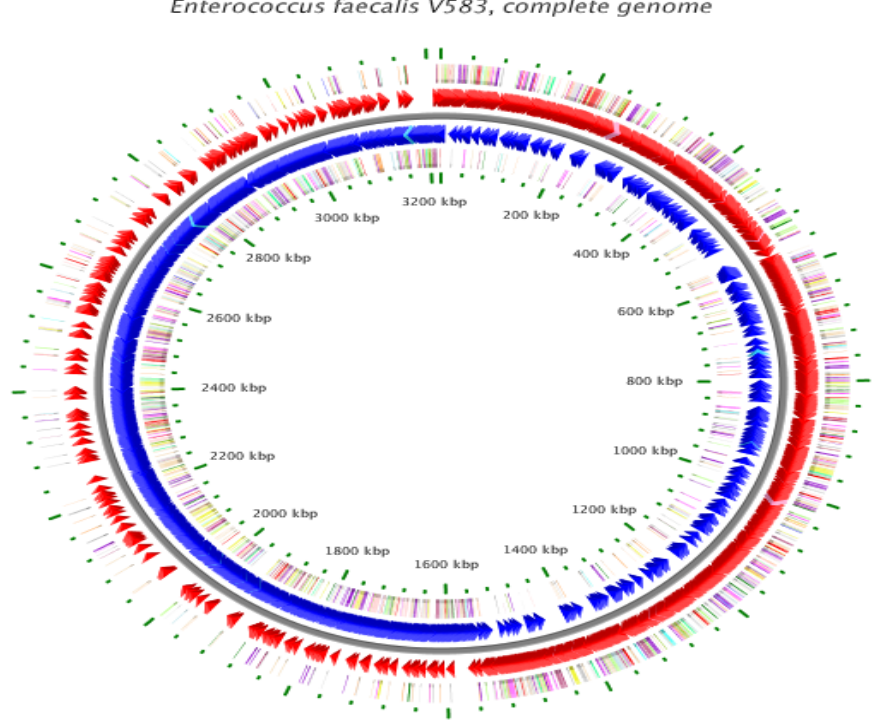

Accession: NC_004668

Length: 3,218,031 bp; Genes: 3,193

Figure 2: Enterococcus faecalis V583, complete genome sequencing

Source: Stothard Paul, Van Domselaar G, Shrivastava S, Guo A, O'Neill B, Cruz J, Ellison M, Wishart DS (2005) BacMap: an interactive picture atlas of annotated bacterial genomes. 


\section{Cell Metabolism}

Enterococci are facultative anaerobes. They have a fermentative metabolism in which they can convert carbohydrates to lactic acid. They are usually considered strict fermenters because they lack a Kreb's cycle respiratory chain. E. faecalis are capable of not only fermentation to produce lactic acid but also can "catabolize a spectrum of energy sources from carbohydrates, glycerol, lactate, malate, citrate, diamino acids and manya-keto acids" (Ceciet al., 2015).

\section{Epidemiology of Vancomycin} ResistantEnterococcus faecalis

The epidemiology of VRE has not been elucidated completely; however, certain patients populations are at increased risk for VRE infection or colonization. These include critically ill patients or those with severe underlying disease or immunosuppression, such as ICU patients or patients on oncology or transplantation wards, those who have had an intra-abdominal or cardiothoracic surgical procedures, those with an indwelling urinary or central venous catheter, and those who have had a prolonged hospital stay or received multiple antimicrobial agents, hospital Infection Control Practices Advisory Committee (HICPAC) published recommendations in February 1995 (CDC, 2017), These recommendations mainly focused on (i)prudent use of vancomycin, (ii)education of hospital staff, (iii)effective use of microbiology laboratory, and (iv) implementation of infection control measures. Enterococci have emerged as one of the leading causes of health care-associated infections (Chuang et al., 2010). The increase in antibiotic resistance among Enterococci, specifically to vancomycin, has become a major clinical and epidemiological problem (Coombs et al., 2014). UK, France, Turkey and Malaysia are among the many countries that have reported infection or colonization with VRE, and the spectrum of documented infections includes endocarditis, thrombophlebitis and bacteraemia. At the Detroit Medical Centre (DMC), located in southeastern Michigan, vancomycin-resistant $E$. faecalis (VR E. faecalis) is unusually common. More than $38 \%$ of vancomycin-resistant enterococci (VRE) at DMC were E. faecalis in 2009 (Hayakawa et al., 2011), in contrast to the national prevalence of $11.7 \%$ (Shioya et al., 2011), and the prevalence of VRE. faecalis has been growing (Hayakawa et al., 2011). A recent study of skilled nursing facilities in southeastern Michigan also reported a high prevalence of VR E. faecalis, which accounted for $52 \%$ of total VRE isolates in the study
(Flannery et al., 2011; CDC, 2017). According to recent National Nosocomial Infection Surveillance (NNIS) surveys, enterococci remain in the top 3 most common pathogens that cause nosocomial infections. $E$ faecalis frequently cause UTIs, bloodstream infections, and wound infections in hospitalized patients in the United States and in South Western Nigeria (Kumurya and Yahaya, 2016; CDC, 2017). Nosocomial enterococcal infections typically occur in very ill debilitated patients who have been exposed to broad-spectrum antibiotics (Jayne, 2017; Susan, 2018). The increased prevalence of serious $E$ faecalis infections has been associated with the rise/use of third-generation cephalosporins (Dance, 2013; Anderson et al., 2015). In 1989, VRE was first reported in New York City; subsequently, VRE has spread rapidly throughout the United States. From 1989-1993, the NNIS surveys reported that the percentage of enterococcal isolates exhibiting vancomycin resistance increased from $0.3 \%$ to $7.9 \%$, with a 34-fold rise seen in ICUs. In 2003, the rate of nosocomial enterococcal isolates showing vancomycin resistance in ICU patients increased to more than $28 \%$-an increase of $12 \%$ compared with 1998-2002. NNIS data reveal the pooled mean for VRE species from all ICUs, non-ICU inpatient areas, and outpatient areas were $13.9 \%, 12 \%$, and $4.6 \%$, respectively, from 1998 through June 2004. VRE was initially isolated mainly in large university hospitals ( Susan, 2018) Still subsequent reports demonstrate the presence of significant VRE epidemics in community hospitals and chronic care facilities, whereby a single clone can easily spread(Arias et al., 2010; Sinelet al., 2017).

In contrast, Europe appears to have a large community reservoir of VRE without as rapid an increase in incidence of hospital-associated infections seen in the United States. In European countries, vanA-type VRE has been isolated from various farm animals, chicken carcasses, other meat products, and wastewater samples from sewage treatment plants. In 1994, a German community screened 100 healthy people for VRE, and $12 \%$ were found to be carriers (CDC, 2017; Jayne, 2017). In Europe, the use of avoparcin, a glycopeptide antibiotic, as a growth promoter for farm animals has been proposed to explain the epidemiology of VRE. Until banned by the European Union in 1997, avoparcin had been used in several European countries and provided a selective pressure for the emergence and spread of vancomycin-resistant genes. This hypothesis is supported by a Danish study that found vanA-type VRE in chicken stool samples from farm using avoparcin, not in 
samples from farms not using avoparcin (Susan, 2017).

Among the Saxony-Anhalt region in Germany, the prevalence of VRE fecal colonization in healthy individuals after discontinuing avoparcin use in animal husbandry decreased from $12 \%$ to $3 \%$, concurrent with a similar decrease in the prevalence of VRE in German poultry products (Puzukiet al., 2014).

A Korean study documented unexpectedly high resistance levels in VRE isolates to daptomycin, linezolid, and tigecycline despite the occasional use of these antibiotics in Korean hospitals (Jaremkoetal., 2013; CDC, 2017).

Distributions of Vancomycin Resistant Enterococcus

Enterococcus faecalis infections are more common in elderly patients because of various associated factors that are more common in these patients (Jayne, 2017). For example, urinary tract catheterization and instrumentation are more common in elderly populations. Abdominal surgery for diverticulitis or biliary tract disease is also performed more commonly in elderly persons. In a recent series, most cases of enterococcal endocarditis occurred in elderly individuals. In neonates, enterococci occasionally cause bacteremia and meningitis. Outbreaks of enterococcal infections, including VRE infections, have been reported in neonatal ICUs, pediatric ICUs, and hematology/oncology units (Susan, 2017). Overall, VRE infections are less common in pediatric patients than in adults (Flannery et al., 2011; Susan, 2017). In general, Enterococcus faecalis infections are distributed equally between the sexes. Although UTIs are more common in healthy women than in healthy men, enterococci are an uncommon cause of uncomplicated cystitis in this setting. In published series of enterococcal endocarditis, men often outnumber women (Asgharzadehand Kafil, 2014; Susan, 2017).

Mechanism of Vancocmycin Resistance in Enterococcus faecalis

Vancomycin is a bactericidal drug that functions by binding to the terminal d-Ala-d-Ala in the pentapeptide portion of the $\mathrm{N}$ acetylglucosamine (NAG)- $\mathrm{N}$-acetylmuramic acid (NAM) peptidoglycan (PG) cell wall precursor, resulting in reduced integrity and, ultimately, cell death. Resistance to glycopeptides in Enterococcus spp. is mediated by the vancomycin resistance (van) operon. This operon may be carried chromosomally or extrachromosomally on a plasmid. The van operon consists of vanS-vanR, a response regulator; van $\mathrm{H}$, a d-lactate dehydrogenase gene, vanX, and a variable ligase in which 9 variant genes have been identified (vanA, vanB, vanC, vanD, vanE, vanG, vanL, vanM, and vanN) (Ceciet al., 2015). Two of these (vanA and vanB) are mediated by newly acquired gene clusters not previously found in enterococci ( Cetinkaya, 2000). vanB resistance phenotypes were described primarily in $E$. faecalis and $E$. faecium(CDC, 2017). Expression is inducible by the two-component system (TCS)vanS/R, which senses disruptions in the cellular membrane caused by glycopeptides, as well as cell wall damage caused by bacitracin or polymyxin B (CDC, 2012). The variable ligase gene is central in determining the level of vancomycin resistance (low, medium, or high), with the most commonly identified genes being vanA, vanB, and vanC. vanA is plasmid borne, confers high-level resistance (MIC, $>256 \mu \mathrm{g} / \mathrm{ml}$ ) to vancomycin, and is most commonly associated with $E$. faecium and $E$. faecalis, while chromosomally encoded vanC confers low-level resistance (MIC, 8 to $32 \mu \mathrm{g} / \mathrm{ml}$ ) to vancomycin and is almost exclusively found in $E$. gallinarum, E. casseliflavus, and $E$. flavescens. Finally, vanZ, which is present on vanA-carrying strains, confers modest resistance to teicoplanin through an unknown mechanism. Differences in the level of resistance are likely a result of pentapeptide composition, as the ratio between pentapeptides consisting of high affinity to low affinity to vancomycin correlate with the isolate MICs. High-level resistance (HLR) occurs when pentapeptides are mostly composed of low-affinity molecules, and moderate-level resistance (MLR) involves moreheterogeneous pools of high- and low-affinity pentapeptides (Sinel et al., 2017).

\section{vanA resistance}

The vanA cluster is the most common mediator of vancomycin resistance in enterococci and its expression is under the regulation of two promoters. The first is responsible for the transcription of vanS/R, the TCS that regulates vanA expression and function. The system's sensor is vans, a transmembrane protein with a histidine kinase domain that responds to the presence of glycopeptides by phosphorylating the response regulator vanR (Asgharzadeh and Kafil, 2014). Once activated, and bound to the second promoter region, located upstream of the resistance genes, activating their transcription. The first step in expressing vancomycin resistance is the transcription of vanH that encodes a dehydrogenase, which allows for $D$-lactate production from pyruvate. The following gene, vanA, produces a ligase that enables the addition of D-Lac to D-Ala before adding it to a tripeptide precursor. 
The resulting pentapeptide is incorporated into the growing cell wall and allows for crosslinking of the peptidoglycan structure. vanX, a D,D-dipeptidase, and many, a DdcY, work to clear the usual D-Ala-D-Ala dipeptides (which will bind Vancomycin if incorporated in the cell wall) and the standard D-Ala-ending pentapeptide chains from the pool of cell wall precursors, respectively. Thus, the destruction of D-Ala-ending pentapeptide precursors is crucial for the mechanism of glyco-peptide resistances. A gene, designated vanZ, encodes for a putative protein whose function has not been completely elucidated, but that was shown to confer teicoplanin resistance when expressed independently in an $E$. faecium strain (Shioya et al., 2011).

\section{vanB resistance}

Isolates carrying vanB are less prevalent than vanA-carrying strains, but can be found throughout the world and are commonly identified in Australia, where the majority of $E$. faecium VRE isolates carry vanB(Coombs et al., 2014), as with vanA, Resistance in van $B$ is mediated by converting d-Ala-d-Ala to d-Ala-dLac. However, vanB confers varied Resistance to Vancomycin, ranging from moderate- to high-level resistance (MIC range, 4 to >256 $\mu \mathrm{g} / \mathrm{ml}$ ) (Lebreton et al., 2013). Resistance to vancomycin is proportional to the percent composition of d-Ala-d-Lac to d-Ala-d-Ala (Coombs et al., 2014). Smaller amounts of $d$ Ala-d-Lac incorporation might result from reduced expression of the vanB operon, a reduction in vanX or vanB enzymatic activity, or a combination of minor mechanical changes. Teicoplanin resistance is not observed in vanBcarrying isolates, as vanZ is not encoded in this operon (Leonard, 2017).

The vanG resistance type

$E$. faecalis possessing a vanG cluster were lowlevel vancomycin-resistant and Teicoplanin susceptible (Arias et al., 2010). Resistance ismediated via inducible synthesis of D-Ala-DSer-terminated cell wall precursors. Only fewisolates have been described and vangene clusters identified allow differentiation into two subtypes. According to its order and gene composition, the chromosomal vanG cluster consists of seven genes that appear to be reassembled from different van operons. In contrast to all the other van operons, the vanecluster encodes three putative gene products with acquisition of the vanG cluster was associated with a transfer of a $240 \mathrm{~kb}$ chromosome fragment flanked by imperfect inverted repeats (Sinel et al., 2017). Crystallisation and X-ray analysis of the vanG D-
Ala-D-Ser ligase complex with ADP was described recently (CDC, 2017).

The vanL resistance type

A single $E$. faecalisisolate from Canada (N060364) expressed low- level vancomycin resistance by a new mechanism called vanL (CDC, 2017). The corresponding vanL gene mediates D-Ala-D-Ser ligation. The vanLgene cluster was similar in organization to the vanCoperon, but the vanT serine racemase was encoded by two separate genes, vanTmL (membrane binding) and vanTrL (racemase) resembling the two functional domains of the otherwise combined vanT type racemase(Lebretonet al., 2013).

Transmission of Vancomycin- Resistant Enterococci

Vancomycin Resistant Enterococci (VRE) transmission by health care workers whose hands become transiently contaminated with the organism while caring for infected patients, probably the most common mode of nosocomial communication. This mode of transmission is seen by the recovery of VRE and other resistant enterococci from cultures of specimens fromhealth care workers' (Cetinkaya, 2000; Leonard, 2017).VancomycinResistant Enterococci transmission may also occur by way of contaminated medical equipment, although this is probably much less important than transmission by the hands of personnel. Electronic thermometers contaminated with outbreak strain were epidemiologically implicated in an outbreak (Cetinkaya, 2000; Leonard, 2017).

Vancomycin Dependent Enterococci (VDE)

An interesting phenomenon that has developed in some strains of vanA-and vanB type VRE is that of vancomycin dependence (Sinel et al., 2017). These enterococci not just resistant to vancomycin but now require it for growth vancomycin dependent enterococci have been recovered from apparently culture- negative clinical samples by plating them onto vancomycin-containing agar such as that used for campylobacter or gonococci. A likely explanation for the phenomenon of vancomycin dependence is that these enterococci turn off their normal production of D-Ala-D-Ala and grow only if a substitute dipeptide-like structure is made (Cetinkaya, 2000; Dubin and Pamer, 2014).

Prevention and Control of Vancomycin Resistant Enterococci (VRE)

In order to reduce nosocomial transmission of Vancomycin Resistant Enterococci to its barest minimum, hospitals must use multidisciplinary approach that requires participation by a variety of departments and personnel 
(Cetinkaya, 2000; CDC, 2017). Antimicrobial stewardship (AMS) is fundamental in the control of major hospital pathogens. In particular, restriction of the use of extended- spectrum cephalosprins, quinolones is of proven worth for VRE(Cetinkaya, 2000; Dance, 2013).

\section{CONCLUSION}

Vancmycin Resistant Enterococci (VRE) is a gram-positive commensal bacterium that inhabits the gastrointestinal tracts of humans and other mammals, however, it can cause lifethreatening infections in humans, especially in

\section{REFERENCES}

Aliya, E., Jawwad, I. A., Hina, A. and Muhammed, Y. (2021). Dental Caries and the influence of Associated Factors- A cross sectional study. Journal of Evolutionary Medicine and Dental Science.10 (21): 1616-1620

Anderson, A.C., Jonas, D., Huber, I., Karygianni, L., Wolber, J. and Hellwig, E. (2015). Enterococcus faecalis from food, clinical specimens, and oral sites: Prevalence of Virulence Factors in Association with Biofilm Formation. Front Microbiology. 6:1534

Arias, CA.; Contreras, GA.; Murray, and BE. (Jun 2010). "Management of multidrugresistant enterococcal infections". Clin Microbiol Infect.16 (6): 555-62. doi:10.1111/j.1469-0691.2010.03214.x. PMID 20569266.

Asgharzadeh, $M$ and Kafil, H. S. (2014).Vancomycin-resistant

Ennterococcus faecalis isolated from Education Hospital of Iran. Journal of Clinical Medicine:9(4) 323-327

Ceci, M., Delpech,G., Sparo, M.,Mezzina, V., Sanchez Bruni, S.and Baldaccini, B. (2015). Clinial and microbiological features of bacteremia caused by Enterococcus faecalis. Journal for Infective Device Countries. 9 (11):1195203

Cetinkaya, Y. Falk, P. and Mayhall, C. G. (2000). Vancomycin-Resistant

Enterococci. Clinical Microbiology Review.13 (4): 686-707

Center for Diseases Control. (2017) about antimicrobial resistance- Antiviotic/ antimicrobial resistance

Centers for Disease Control (2012). CDC reminds clinical laboratories and healthcare infection preventionists of their role in the search and containment of vancomycin-resistant Staphylococcus aureus (VRSA).Centers the nosocomial environments, where there are naturally high level of antibiotic resistance and the very ill debilitated patients who have been exposed to broad spectrum antibiotics, and the immune-compromised are found. This review emphasizes the nosocomial nature of VRE infections with their tendency to become endemic given the reasons mentioned above, yet vancomycin still continues to be the drug of choice for serious life threatening infections as sepsis, pneumonia and endocarditis caused by Enterococcus faecalis.

for Disease Control and Prevention, Atlanta,

GA.http://www.cdc.gov/HAl/settings/l ab/vrsa_lab_search_containment.html.

Chuang, Y.C., Wang, J.T., Chen, M.L. and Chen, Y.C. (2010). Comparison of an automated repetitive sequence-based PCR microbial typing system with pulsed field gel electrophoresis for molecular typing of vancomycinresistan Enterococcus faecium .Journal of Clinical Microbiology; 48:2897-2901 doi: $\quad 10.1128 / J C M .00136-10$ pmid:20554812 15.

Coombs,G.W., Pearson, J.C., Daley, D.A., Le,T., Robinson, O.J., Gottlieb, T., Howden, B.P., Johnson, P.D., Bennett, C.M., Stinear,T.P. andTurnidge,J.D. ( 2014) Australian Group on Antimicrobial Resistance. Molecular epidemiology of enterococcal bacteremia in Australia.Journal Clinical Microbiology 52:897-905.10.1128/JCM.03286-13.

Dance, S. (2013). Approaching zero: temporal effects of a restrictive antibiotic policy on hospital acquired Clostridium defficle, extended spectrum Blactamase producing coliforms and methicillin Staphylococcus aureus. .International Journal Antimicrobial Agents;41:137-

142.doi:10.1016/ijanticag,201210.013.

Dubin, K. and Pamer, E. G. (2014).Enterococci and their interactions with the intestinal mirobiome. Microbiology Spectrum: 5 (6) 6-12

Flannery, E.L., Wang, L., Zollner, S., Foxman, B., Mobley, H.L. andMody, L. (2011). Wounds, functional disability, and indwelling devices are associated with cocolonization by methicillin-resistant Staphylococcus aureus and vancomycinresistant enterococci in southeast Michigan. Clinical Infectious Disease. 53:1215-1222. 
Hayakawa, K., Marchaim, D., Vidaillac, C., Lephart, P., Pogue, J.M., Sunkara, B., Kotra, H., Hasan, A., Shango, M., Yerramalla, Y., Osunlana, A.M., Chopra, T., Dhar, S., Salimnia, H., Rybak, M.J. and Kaye, K.S. (2011).Growing

prevalenceofvancomycin-resistant

Enterococcus faecalisin the region with the highestprevalence of vancomycinresistant Staphylococcus aureus.Infection.ControlHospital Epidemiology. 32:922-924.

Jayne, L. (2017). What's to know about Enterococcus faecalis? Medical News Today July 12, 2017.

Kumurya, A. S. and Yahaya, H. (2016) Detection of vancomycin resistance rate among Methicillin-resistant Staphylococcus aureusisolates in Kano, Nigeria. Accesss Journal of Microbiology: 2(1)7-12

Lebreton, F. vanSchaik, W. and McGuire, A. M (2013). Emergence of Epidemic Multidrug-resistant Enterococcus faecium from Animal and commensal strains. Microbiology. 4:534-613

Sinel, C. Augagneur, Y. Sassi M. Bronsard, J. Cacaci, M. Guerin, F. Sanguinetti, M. Meignen, $P$. and Cattoir, V. (2017).Small RNAs in vancomycinresistant Enterococcus faecium involved in daptomycin response and resistance. Science reports 7(1): 11067

Susan, L.F. (2018). Enterococcal infections Medscape (emedicine, Medscape. Com)

Zhu, W., Murray, P.R., Huskins, W.C., Jernigan, J.A., McDonald, L.C., Clark,N.C., Anderson, K.F., McDougal, L.K., Hageman, J.C., Olsen-Rasmussen, M.,Frace, M., Alangaden, G.J., Chenoweth, C., Zervos, M.J., RobinsonDunn, B., Schreckenberger, P.C., Reller, L.B., Rudrik,J.T. and Patel, J.B.(2010). Dissemination of an enterococcus Inc18like vanAplasmid associated with vancomycin- resistant Staphylococcus aureus. Antimicrobial Agents Chemotherapy. 54:4314-4320. 